\title{
The Cerebral Cavernous Malformation signaling pathway promotes vascular integrity via Rho GTPases
}

\author{
Kevin J. Whitehead ${ }^{1,2}$, Aubrey C. Chan ${ }^{2}$, Sutip Navankasattusas ${ }^{2}$, Wonshill Koh ${ }^{3}$, Nyall R. \\ London ${ }^{2}$, Jing Ling ${ }^{2}$, Anne H. Mayo ${ }^{3}$, Stavros G. Drakos ${ }^{2}$, Christopher A. Jones ${ }^{2}$, Weiquan \\ Zhu $^{2}$, Douglas A. Marchuk ${ }^{4}$, George A. Davis ${ }^{3}$, and Dean Y. Li ${ }^{1,2}$ \\ ${ }^{1}$ Division of Cardiology, Department of Medicine, 30 N. 1900 East, Room 4A100 SOM, University \\ of Utah, Salt Lake City, UT 84132 \\ ${ }^{2}$ Molecular Medicine Program, 15 N. 2030 East, Room 4140, University of Utah, Salt Lake City, \\ UT 84112 \\ ${ }^{3}$ The Department of Medical Pharmacology and Physiology, School of Medicine University of \\ Missouri School of Medicine, MA415 Medical Sciences Building, One Hospital Drive, Columbia, \\ MO 65212 \\ ${ }^{4}$ Department of Molecular Genetics and Microbiology, Box 3175, Duke University Medical Center, \\ Durham, NC 27710
}

\section{SUMMARY}

Cerebral cavernous malformation (CCM) is a common vascular dysplasia that affects both systemic and CNS blood vessels. Loss of function mutations in the CCM2 gene cause CCM. Here we show that targeted disruption of $\mathrm{Ccm} 2$ in mice results in failed lumen formation and early embryonic death through an endothelial cell autonomous mechanism. We demonstrate that CCM2 regulates endothelial cytoskeletal architecture, cell-cell interactions and lumen formation. Heterozygosity at $C \mathrm{~cm} 2$, a genotype equivalent to human CCM, results in impaired endothelial barrier function. Because our biochemical studies indicate that loss of CCM2 results in activation of RHOA GTPase, we rescued the cellular phenotype and barrier function in heterozygous mice using simvastatin, a drug known to inhibit Rho GTPases. These data offer the prospect for pharmacologic treatment of a human vascular dysplasia using a widely available and safe drug.

\section{INTRODUCTION}

Cerebral cavernous malformations (CCM) are common vascular malformations that affect the systemic and CNS vasculature with a prevalence of 1:200-2501,2 in unselected populations. CCM consist of enlarged microvascular channels lined by a single layer of endothelium without smooth muscle support. Those who harbor these vascular lesions are

\footnotetext{
Users may view, print, copy, and download text and data-mine the content in such documents, for the purposes of academic research, subject always to the full Conditions of use:http://www.nature.com/authors/editorial_policies/license.html\#terms

Corresponding Author: Dean Y. Li, Building 533 Room 4220, 15 N 2030 East, Salt Lake City, UT 84112, dean.li@ hmbg.utah.edu. Supplementary information is available on the Nature Medicine website.

Competing interests statement: the authors declare competing financial interests.
} 
subject to an unpredictable risk of hemorrhage for which no pharmacologic therapy currently exists 3 . Even prior to overt hemorrhage, all lesions are surrounded by hemosiderin, the iron-laden deposits that result from extravascular blood that can be sensitively detected by MRI4 and suggest abnormal endothelial barrier function5. Although lesions have been described in a variety of vascular beds6, clinical manifestations are most common in the CNS, where the consequences of leak and hemorrhage can be stroke, seizure, or even death. A large proportion of cases are familial, following an autosomal dominant inheritance pattern. Genetic studies have shown that heterozygous loss of function mutations in CCM2 also known as Osmosensing scaffold for MEKK3 (OSM), Malcavernin, or MGC4607 - cause cavernous malformations7,8. Two other structurally unrelated genes, KREV1 interaction trapped-1 (KRIT1) - also known as CCM19,10 - and Programmed cell death 10 (PDCD10) also known as CCM311 - have also been associated with CCM. Even with the hindsight afforded by the human genetic studies, there is little to recommend these genes as obvious candidate genes for any vascular disease.

CCM2 (OSM) was identified in a screen for genes involved in the cellular response to osmotic shock12. These stress-activated pathways include a signaling cascade involving Rho-family GTPases such as RHOA, RAC1 and CDC42, and MAP kinases such as p38 and JNK. In fibroblasts, CCM2 (OSM) was required for the assembly of a complex between the kinase MEKK3 and its substrate MKK3 to phosphorylate p38 in response to signaling from the GTPase RAC1.

Efforts to incorporate these biochemical insights into a molecular model to explain the pathogenesis of CCM have been plagued by controversy over the cell specificity of the CCM2 requirement. Though a compromised vasculature defines CCM, there are compelling data that suggest a neuronal role in the etiology of the disease. First, the expression of the three genes involved in CCM is neither specific nor selective for the endothelium in culture or in animal models13-15. Second, there is precedence that neural deletion of Alpha-V integrin disrupts the cerebral vasculature and causes hemorrhage in mice16. Conversely, it has been observed that KRIT1 can act as an effector of the small GTPase RAP1A in cultured endothelial cells17. The tissue specificity of $\mathrm{Ccm} 2$ function remains unknown.

In this manuscript we demonstrate that $C \mathrm{~cm} 2$ is required for the first essential angiogenic event during development, the formation of the first branchial arch artery. We also show by tissue specific ablation of $\mathrm{Ccm} 2$ that vascular defects associated with $\mathrm{Ccm} 2$ mutations are endothelial autonomous. Cultured endothelial cells with reduced $C C M 2$ expression have intrinsic impairment of lumen formation and bear many hallmarks of RHOA GTPase activation, including functional, morphologic, and biochemical changes. Furthermore, CCM2 is shown to bind to RHOA, and Rho inhibition can reverse many of the endothelial changes. Decreased endothelial barrier function is observed in cultured cells and in mice with heterozygous mutations of $\mathrm{Ccm} 2$, which genocopy human CCM. Impaired barrier function can be rescued in vitro by RHOA inhibition, and in vivo by pre-treatment with simvastatin, an indirect inhibitor of Rho GTPases. This work demonstrates that CCM2 is critical for vessel integrity and modulates RHOA signaling. To the extent that this pathway also leads to vascular dysplasia in human CCM, our studies open up the possibility of targeted pharmacologic therapies to address vascular defects in this common condition. 


\section{RESULTS}

\section{Ccm2 is required for angiogenesis}

A putative null allele of $C \mathrm{~cm} 2$ was identified from a collection of gene trap induced mutations (Bay Genomics consortium). This allele has been termed Ccm2 $2^{G t(p G t 0 L x f) 1 D m a r}$ (hereafter designated $\mathrm{Ccm}^{\text {tr }}$ ), and consists of the gene trap vector inserted into exon 6 of $C \mathrm{~cm} 2$ along with a 45 nucleotide deletion of genomic sequence14, disrupting transcription of $\mathrm{Ccm} 2$ (Supplementary fig. 1a-c). Mice heterozygous for $\mathrm{Ccm} 2^{t r}$ are viable and fertile as previously reported 14 . We observed no homozygous mutant mice at weaning. We identified mutant embryos in Mendelian ratios until the ninth day postcoitum (E9.0). Starting at E9.0 we began to recognize a gross phenotype in mice lacking $C \mathrm{~cm} 2$ (Supplementary table 1). The homozygous mutant embryos failed to organize the yolk sac vasculature and showed evidence of growth arrest at E9.0. Pericardial effusions subsequently developed prior to embryo resorption at E11.5. No viable mutants were observed at E9.5 or beyond. The timing of death in these embryos is consistent with failed angiogenesis.

We studied embryos at E8.5 before the mutant phenotype could be grossly detected. Embryos were stained with antibodies against the endothelial cell surface protein CD31 (PECAM) or alpha-smooth muscle actin and observed by whole mount confocal immunofluorescent microscopy or sectioned and studied by immunohistochemistry. The initial patterning of the dorsal aorta (Supplementary fig. 1d, e) and yolk sac primary vascular plexus (data not shown) by vasculogenesis 18 is intact in mutants. Heart development is also normal (data not shown). After the initial vascular pattern is established, however, profound defects occur in the development of subsequent vessels by angiogenesis (Fig. 1a, b). The first defects observed in mutant embryos included abnormalities of the first branchial arch artery and the intersomitic arteries at E8.5 (Fig. 1a, b and Supplementary fig. 1e, f). The first branchial arch artery, required to connect the dorsal aorta to the heart, fails to form a proper lumen in mutants. Adjacent portions of the aorta are also narrow and irregular, whereas the previously normal caudal portion of the dorsal aorta becomes enlarged (Supplementary fig. 1d). Yolk sac vascular remodeling is abnormal (data not shown). The failure of the branchial arch arteries has profound physiologic consequences on the embryo. In vivo ultrasound studies demonstrate that despite normal frequency of cardiac contractions, circulation is not established in homozygous mutants (Fig. 1c and Supplementary movies 1 and 2). Branchial arch artery failure is not confined to the arteries of the first arch. The second and third pair of branchial arch arteries should normally form by E9.5. We injected India ink into the ventricles of mutant embryos at E9.5 and did not observe passage of ink into the dorsal aorta of mutants (Fig. 1d). Unlike the anterograde flow observed in wild type littermates, ink passed retrograde from the ventricle through the atrium and into the common cardinal vein in mutant embryos. Growth arrest and embryonic death resulted from failed circulation at E8.5.

\section{Ccm2 is required in the endothelium}

Mice with gene trap mutations in $C \mathrm{~cm} 2$ establish an essential role for this protein in angiogenesis. This mutation is present in all cells of the embryo and thus does not distinguish which tissues require $C \mathrm{~cm} 2$ for normal function. To determine tissue specificity, 
we developed mice with a conditional mutation in $C \mathrm{~cm} 2$ using CRE-Lox technology (Supplementary fig. 2). This allele is termed $C \mathrm{~cm} 2^{t m l K w h i}$ (hereafter referred to as $C \mathrm{~cm} 2^{f l}$ ). The $C c m 2$ gene remains intact until the allele is exposed to CRE recombinase which deletes exons 3-10 of $C \mathrm{~cm} 2$. Mating $C \mathrm{~cm} 2^{f / /+}$ mice with HPRT-CRE mice19 resulted in a new, heritable mutant allele termed $\mathrm{Ccm} 2^{t m 1.1 K w h i}$ (hereafter referred to as $\mathrm{Ccm}^{-}$). Homozygous mutant $\left(\mathrm{Ccm}^{-{ }^{--}}\right)$mice phenocopy the gene trap $\left(\mathrm{Ccm} 2^{\mathrm{tr} / \mathrm{tr}}\right)$ mutants (Supplementary fig. 2e). CRE recombinase can also be expressed in a tissue specific manner, under the control of a variety of promoters. We subsequently examined a number of tissue-restricted, somatic mutants for defects in angiogenesis. Mice lacking $\mathrm{Ccm} 2$ in the endothelium $\left[C \mathrm{Cm} 2^{f l /} ; \operatorname{Tg}(T i e 2-C R E)\right] 20$ resemble germline mutants with similar vascular defects and timing of embryonic death (Table 1 and Fig. 2). Endothelial cell specific deletion of $\mathrm{Ccm} 2$ is uniformly lethal in development.

The expression of $\mathrm{Ccm} 2$ in neural tissues and the predominance of CCM lesions in the central nervous system suggest a possible role for $\mathrm{Ccm} 2$ in neural cells. Mice lacking $\mathrm{Ccm} 2$ in neural tissues were generated with CRE driven by a Nestin promoter $\left[\mathrm{Ccm} 2^{f l /-} ; \mathrm{Tg}(\mathrm{Nes}-\right.$ $C R E)] 21$. These mutants had no defects in angiogenesis at E9.0 and were found in expected ratios at birth (Table 1 and Fig. 2). Another important contributor to the milieu of endothelial cells in vivo is the smooth muscle cell. Mice lacking $C \mathrm{~cm} 2$ in smooth muscle cells were generated with a Transgelin-CRE $\left[C \mathrm{Cm} 2^{f l /-} ; \operatorname{Tg}(\mathrm{Tag} \ln -\mathrm{CRE})\right] 22$. Smooth muscle mutants were also found at birth, with normal vasculature at E9.0 (Table 1 and Fig. 2). These data indicate an essential role for $C \mathrm{~cm} 2$ in endothelial cells for the initial events of angiogenesis.

\section{CCM2 regulates lumen formation via the actin cytoskeleton}

Our genetic studies led us to evaluate the function of CCM2 in endothelial cells. We observed CCM 2 expression by real time quantitative RT-PCR in all endothelial cell types studied. A single siRNA construct was able to decrease CCM2 transcripts by $80-90 \%$ in human microvascular (dermal) endothelial cells (HMVEC) and human umbilical vein endothelial cells (HUVEC) (Fig. 3a).

Endothelial cells in three-dimensional culture spontaneously develop tube-like structures that resemble the microvasculature and model events in developmental angiogenesis 23 . We tested the role of CCM2 in lumen formation in vitro by comparing HUVEC cells treated with CCM2 siRNA with either a luciferase or non-sense control siRNA in a threedimensional assay of tube morphogenesis (Fig. 3b, c and Supplementary movies 3 and 4). Control HUVECs form vacuoles that coalesce into tube-like structures over the course of 24 h, whereas CCM2-depleted HUVECs formed fewer lumens with much smaller lumen crosssectional area (Fig. 3d, e). This defect was observed at the single cell stage prior to the formation of multicellular structures (Fig. 3d). These observations suggest a central and endothelial intrinsic role for $C C M 2$ in the development of precursor vacuoles as well as the coalescence and expansion of these structures to form the vascular lumen. Consistent with this hypothesis, we observed up-regulation of $C C M 2$ mRNA by RT-PCR in a time course parallel with lumen formation in control HUVECs (Fig. 3f). Lumen failure is not a consequence of insufficient endothelial migration, or the ability to form filopodial sprouts. 
HUVECs treated with CCM2 siRNA have increased sprouting of cell processes when initially plated in three-dimensional culture (Fig. 3g). Similarly, HMVECs treated with CCM2 siRNA have increased haptotactic migration (Fig. 3h).

Lumen formation is dependent upon the cellular cytoskeleton24. The CCM2-deficient HMVECs had a striking increase in actin stress fibers traversing the cell, with less cortical actin at the cell periphery (Fig. 4a). Actin redistribution was correlated with a decrease in barrier function and increased permeability of the endothelial monolayer (Fig. 4b, c). We observed decreased electrical resistance and increased transit of macromolecules (HRP) across CCM2-deficient monolayers compared to control.

\section{CCM2 regulates actin and MAPK via RHOA}

The Rho family of small GTPases are important regulators of the cellular cytoskeleton. Impaired lumen formation24, increased actin stress fibers and decreased barrier function 25 in endothelial cells suggest activation of RHOA. Consistent with the cellular phenotype, we observed increased active (GTP-bound) RHOA in CCM2-depleted HMVECs compared to control (Fig. 4d). We found no change in the activation of RAC1 and found less basal activation of CDC42. By immunoprecipitation we found that CCM2 binds to RHOA and RAC1 but not CDC42 (Fig. 4e). Inhibition of RHOA signaling either at the level of RHOA, with $\mathrm{C} 3$ transferase26, or downstream at the level of Rho-Kinase (ROCK), with the ROCK inhibitor Y-2763227, blocked the stress fiber response (Fig. 4f) of CCM2-depleted HMVECs. C3 transferase was also able to significantly rescue barrier function in these cells (Fig. 4g).

CCM2 has also been implicated in MAPK signaling12. We used phospho-specific antibodies to profile the activation state of MAPK families in the absence of CCM2 (Fig. 4h). The main families of MAP kinases are the extracellular signal regulated kinases (ERK) and p38 and JNK, with p38 and JNK also being known as stress regulated protein kinases28. Reduction of CCM2 did not affect the levels of either phosphorylated ERK or p38, but did increase the phosphorylation of JNK and its upstream kinases, MKK4 and MKK7. As GTPases can stimulate MAP kinase signaling, we tested whether increased JNK activation was the result of increased Rho activity by treating cells with the ROCK inhibitor Y-27632. ROCK inhibition decreased the activation of JNK (Fig. 4i). These observations suggest that the loss of CCM2 leads to RHOA activation, causing activation of JNK with an associated change in endothelial phenotype including cytoskeletal changes, impaired lumen formation, and increased migration and vascular permeability.

\section{Simvastatin rescues CCM2 deficiency in vivo}

Humans with CCM have heterozygous mutations in CCM2 and suffer from clinically significant vascular hemorrhage or leakage, not from severe developmental angiogenic defects as observed in mice with homozygous mutations in $\mathrm{Ccm} 2$. To examine the role of $C \mathrm{~cm} 2$ in the disease state, we shifted our attention to mice heterozygous for $C \mathrm{~cm} 2$. We found no difference in vascular patterning (data not shown) or permeability to the intravascular dye, Evans blue (Fig. 5a, saline injections) between heterozygous mice and wild type controls. Clinical reports suggest that physiologic29-31 or genetic32 stressors may 
play a role in disease pathogenesis. An association was observed between accelerated progression of CCM and increasing levels of VEGF29. Consistent with this clinical observation, we observed significantly increased permeability to Evans blue in $\mathrm{Ccm} 2^{+/ t r}$ mice in response to VEGF across a range of doses (Fig. 5a). Increased permeability was also observed in mice with endothelial specific heterozygosity for $\mathrm{Ccm} 2$ (Fig. $5 \mathrm{~b}$ ). These results demonstrate a role for $\mathrm{Ccm} 2$ in the endothelium for the maintenance of normal in vivo barrier function in adults aside from its role in embryonic development.

Our observations in vitro suggested that Rho inhibition might rescue the increased permeability of $\mathrm{Ccm} 2$ heterozygous mice. Unfortunately, mice do not tolerate the ROCK inhibitor Y-27632 (data not shown), and the Rho inhibitor C3 transferase is not useful in vivo. Inhibitors of HMG-CoA reductase (statins) have pleiotropic effects that include the inhibition of Rho GTPases. Simvastatin disrupts the production of key intermediaries in the cholesterol synthesis pathway necessary for RHOA isoprenylation33,34 and has been used as an inhibitor of Rho in vivo35,36. In culture, we found that simvastatin reduced actin stress fibers in both control and CCM2 siRNA treated endothelial cells (Fig. 5c) and decreased the haptotactic migration of CCM2-depleted HMVECs (Fig. 5d). Simvastatin also decreased the phosphorylation of JNK in both control and CCM2 siRNA treated cells (Fig. 5e). In vivo, we found that pretreatment of mice with simvastatin significantly reduced the permeability response of $\mathrm{Ccm}^{+/ t r}$ mice to VEGF with no effect on the induced permeability of $\mathrm{Ccm} 2^{+/+}$ mice (Fig. 5f). These data suggest that abnormal Rho GTPase activity observed in cells depleted of CCM2 is also present in mice with reduced $C \mathrm{~cm} 2$.

\section{DISCUSSION}

Here, we provide the first in vivo evidence that any of the genes causing CCM are required in the endothelium. Previously, the cellular basis of CCM disease was not clear. The extensive neural expression13-15, the CNS predilection of lesions, and lessons learned from Alpha-V integrin targeted mice16 suggested that CCM might be caused by an intrinsic neural cell defect. We demonstrated that mice lacking $\mathrm{Ccm} 2$ die in midgestation from failed angiogenesis and that these vascular defects are endothelial autonomous. Using two distinct mutations we have shown that $\mathrm{Ccm} 2$ is not required for the initial differentiation of endothelial cells from angioblast precursors (vasculogenesis) 18, but rather, that subsequent vessel formation and remodeling (angiogenesis) is impaired at the earliest possible stages. Branchial arch arteries, the first essential angiogenic vessels, fail to form a stable lumen. As a result, the heart is not functionally connected with the vasculature and circulation fails to initiate. Subsequent growth and development of the embryo is severely impaired, and death ensues. Tissue specific mutants have shown that the selective endothelial deletion of $\mathrm{Ccm} 2$ is sufficient to reproduce the vascular defects, whereas mice lacking $C \mathrm{~cm} 2$ in neural or smooth muscle cell lines develop normally. Thus, $\mathrm{Ccm} 2$ is required in the endothelium for angiogenesis.

The recognition of essential endothelial autonomous functions for $\mathrm{Ccm} 2$ allowed us to examine previous biochemical studies in a new light. Previous studies in fibroblasts and other non-endothelial cell types suggested a role for CCM2 as a scaffold tying RAC1 GTPase activity to p38 activation in response to osmotic stress12. Endothelial cells face 
stressors in the context of a monolayer of interacting cells. In addition to the maintenance of individual cell size and shape in the face of stress, endothelial cells must also maintain contact and barrier function with adjacent cells in the monolayer. One might expect that $C C M 2$, as an important molecule in response to stress, would play a role in maintaining stable endothelial cytoskeletal architecture and cell-cell interactions. Our observations in CCM2-depleted HMVECs support this hypothesis. We observe a loss of cortical actin with increased actin stress fibers and decreased endothelial barrier function, changes that are typical of activated RHOA GTPase25, and which can be reversed by inhibitors of RHOA signaling.

Our observations suggest a model whereby there is dynamic equilibrium between vascular stability and instability. The endothelium of a stable blood vessel provides critical barrier functions by controlling fluid, nutrient and cellular transport between intravascular and extravascular compartments. The formation and maintenance of strong cell-cell contacts is a favorable characteristic of endothelial cells in a stable blood vessel. However, when challenged by injury or inflammation the endothelium alters its phenotype and temporarily disrupts cell-cell junctions. In this destabilized state the endothelium can initiate the required migration and angiogenesis responses to the stressor. In this model, CCM2 is required for endothelial cells to assume the stable phenotype, mediated at least in part by limiting RHOA activation24,37,38. Our cell culture data indicate that CCM2 regulates key aspects of the stabilized endothelium, including cellular architecture, barrier function, migration, and tube morphogenesis. Loss of CCM2 favors the destabilized phenotype. Normally, with two functioning alleles of $C C M 2$, the intensity and duration of instability following insult is limited. In our heterozygous mice with only one functioning allele, there is a greater disruption of the stable state with increased permeability in response to VEGF. With the loss of both alleles of $\mathrm{Ccm} 2$ in the endothelium, stability cannot be achieved. Existing vessels enlarge, and exuberant but dysfunctional angiogenic sprouts form, unable to develop stable lumens to allow functional circulation. We speculate that this model also could explain the development of cavernous angiomas in familial CCM. Previously, a "two-hit" hypothesis involving biallelic somatic and germline mutations was proposed to explain the focal nature of vascular lesions in human CCM, supported by a single case report32. The focal loss of the only remaining allele of CCM2 would favor sustained instability of the affected endothelium, resulting in a cavernous angioma. Our in vivo experiments suggest the possibility that the second hit may not be limited to genetic disruptions and could take the form of recurrent exposure to cytokines such as VEGF that are released locally in response to stress or inflammation. The unique importance of strong cell-cell interaction in the central nervous system might render this vascular bed particularly sensitive to such perturbations. The importance of a physiologic response to stress as a second hit could explain the clinical association between elevated levels of VEGF and the rapid progression of CCM29. Thus, in retrospect, a gene originally identified for its role in the cytoskeletal response to stress should have been an obvious candidate gene for human vascular dysplasias. The efficacy of statins in rescuing $C \mathrm{~cm} 2$-related vascular pathology in mutant mice suggests a pharmacologic strategy for treating patients whose therapeutic options are currently limited to brain surgery or radiation. 


\section{METHODS}

Mouse strains

See supplementary methods.

\section{Confocal immunofluorescence of embryos}

See supplementary methods.

\section{Fetal ultrasound}

We studied pregnant mice under isoflurane anesthesia on a heated stage, with continuous monitoring of ECG and respiration. Following laparotomy, embryos were studied within 45 min. The order of embryos was noted for genotype correlation. A Vevo 660 ultrasound machine (VisualSonics) with $40 \mathrm{MHz}$ transducer was used for imaging. We used digital subtraction to illustrate circulating blood in static images (see supplementary methods). Heart rates were determined by M-mode ultrasound.

\section{Ink injection}

We injected India ink as previously described39.

\section{Histology}

See supplementary methods.

\section{Cell culture}

See supplementary methods.

\section{EC vasculogenesis in $3 D$ collagen matrices}

HUVECs (passage 2-5) were suspended within $3.75 \mathrm{mg} \mathrm{ml}^{-1}$ of collagen type I matrices and allowed to undergo EC morphogenesis as described40. Cultures were fixed with $3 \%$ glutaraldehyde for $30 \mathrm{~min}$. Some cultures were stained with $0.1 \%$ toluidine blue in $30 \%$ methanol and destained prior to photography and visualization. Time-lapse microscopy was performed as described41 using a Nikon TE2000U microscope with attached environmental chamber. Time lapse images were examined for total area of both vacuoles and lumens ( $n=$ 5 independent fields), and total process length from all cells ( $n=10$ fields). The number of lumens per field was quantified at $24 \mathrm{~h}(n=3)$. Metamorph (Molecular Devices) software was used to trace and quantify lumen area and process length.

\section{Transfection of ECs with siRNAs}

See supplementary methods.

\section{Reverse transcription-polymerase chain reaction (RT-PCR)}

See supplementary methods. 


\section{Endothelial Cell Haptotaxis}

Haptotactic migration was performed using a modified Boyden chamber assay (Neuro Probe). Polycarbonate membranes ( $8 \mu \mathrm{m}$ pores) were coated with human fibronectin ( $1 \mu \mathrm{g}$ $\mathrm{ml}^{-1}$, Biomedical Technologies, Inc.) on the lower surface. HMVECs were added to the upper well (20,000 cells well $\left.{ }^{-1}\right)$ in EGM-2 and allowed to migrate for $3 \mathrm{~h}$. The membranes were fixed and stained (Hema3 kit, Fisher), non-migrated cells were removed, and the membrane was mounted on a glass slide. The number of migrated cells per high power field was counted for multiple fields and replicates (for control $n=12$ fields, $C C M 2 n=8$ fields). For simvastatin rescue experiments, cells were treated with either $10 \mu \mathrm{M}$ simvastatin (Calbiochem) or carrier only for $24 \mathrm{~h}$ prior to the assay ( $n=3$ fields per condition).

\section{Immunofluorescent Cell Staining}

See supplementary methods.

\section{HRP Permeability}

Transwell inserts (Corning, 48-well, $3 \mu \mathrm{m}$ pore) were coated with human fibronectin and were seeded at 30,000 cells well $^{-1}$. Horseradish peroxidase (HRP $25 \mu \mathrm{g} \mathrm{mL}^{-1}$, Sigma) was added to the top of the insert. Solution from the bottom of the well was assayed $6 \mathrm{~h}$ later for HRP by colorimetric assay. The sample was mixed with guaicol (Sigma) and hydrogen peroxide (Fisher) and measured for absorbance at $490 \mathrm{~nm}$ ( $n=6$ wells each).

\section{Transendothelial Resistance}

An electrode culture array (Applied Biophysics) was coated with human fibronectin and seeded at 50,000 cells well ${ }^{-1}$. Three days after seeding, cells were serum-starved in EBM-2 with $0.2 \%$ bovine serum albumin overnight. Transendothelial resistance was measured using an electric cell-substrate impedance sensing system (ECIS, Applied Biophysics). Cellpermeable $\mathrm{C} 3$ transferase $\left(1 \mu \mathrm{g} \mathrm{ml}^{-1}\right)$ was added to inhibit RHOA. For basal resistance $n=$ 40 wells each; for Rho inhibition experiments, $n=6$ carrier wells each and $n=10 \mathrm{C} 3$ transferase wells each.

\section{GTPase Activation Assays}

See supplementary methods.

\section{Immunoprecipitation}

See supplementary methods.

\section{MAPK Profiling}

See supplementary methods.

\section{Miles assay}

We performed tail vein injections of Evans Blue (0.5\% in normal saline, Sigma) in 8-12 week old mice. Thirty min later either saline or VEGF-165 (R\&D Systems, $10 \mathrm{ng}$ ) was injected in multiple dermal sites. After an additional thirty min, animals were sacrificed, punch biopsies were performed and Evans Blue was eluted from the biopsies in formamide 
(Invitrogen) overnight at $60^{\circ} \mathrm{C}$. The absorbance of Evans Blue was measured at $620 \mathrm{~nm}$, subtracting background absorbance at $740 \mathrm{~nm}$. Simvastatin $\left(20 \mathrm{mg} \mathrm{kg}^{-1}\right)$ was given as an intraperitoneal injection $26 \mathrm{~h}$ before and $2 \mathrm{~h}$ before the intradermal stimuli. For the VEGF dose response experiment $n=5$ mice per group. For the permeability experiment with conditional $\mathrm{Ccm} 2, n=5 \mathrm{Ccm} 2^{+/+}, n=9 \mathrm{Ccm} 2^{f l /-}, n=10 \mathrm{Ccm} 2^{f l /+} ; \mathrm{Tg}(\mathrm{Tie} 2-\mathrm{Cre})$. For the simvastatin experiment $n=3$ mice with control treatment, 4 mice with simvastatin treatment.

\section{Statistics}

For in vitro lumen formation and cell process formation in 3D culture, statistical comparisons between treatment groups were performed with two-tailed Paired Samples Ttest with an alpha value of 0.05 . For transwell in vitro permeability, transendothelial resistance, endothelial cell migration, and the Miles assay of dermal permeability group comparisons were by two-tailed Student's T-test with an alpha value of 0.05 .

\section{Animal Experiments}

All animal experiments were approved by the University of Utah Institutional Animal Care and Use Committee.

\section{Supplementary Material}

Refer to Web version on PubMed Central for supplementary material.

\section{Acknowledgements}

We thank C. Colvin, C. Jones, W. Zhu, and A. Frias for technical assistance; K. Thomas, M. Sanguinetti, S Odelberg and I. Benjamin for critical comments; M. Kahn, M. Ginsberg and R. Stockton for sharing unpublished data; and A. Hall for GTPase cDNA constructs. This work was funded by US National Institutes of Health (K.J.W., D.A.M., G.E.D and D.Y.L.); American Heart Association (K.J.W. and D.Y.L.); HA and Edna Benning Foundation, Juvenile Diabetes Research Foundation, Burroughs Wellcome Fund and Flight Attendants Medical Research Institute (D.Y.L.); and the University of Utah Genetics Training Grant (A.C.C.).

\section{REFERENCES}

1. Otten P, Pizzolato GP, Rilliet B, Berney J. A propos de 131 cas d'angiomes caverneux (cavernomes) du S.N.C. repérés par l'analyse rétrospective de 24535 autopsies. Neurochirurgie. 1989; 35:128131.

2. Robinson JR, Awad IA, Little JR. Natural history of the cavernous angioma. J Neurosurg. 1991; 75:709-714. [PubMed: 1919692]

3. Hasegawa $\mathrm{T}$, et al. Long-term results after stereotactic radiosurgery for patients with cavernous malformations. Neurosurgery. 2002; 50:1190-1197. discussion 1197-1198. [PubMed: 12015835]

4. Chappell PM, Steinberg GK, Marks MP. Clinically documented hemorrhage in cerebral arteriovenous malformations: MR characteristics. Radiology. 1992; 183:719-724. [PubMed: 1584926]

5. Clatterbuck RE, Eberhart CG, Crain BJ, Rigamonti D. Ultrastructural and immunocytochemical evidence that an incompetent blood-brain barrier is related to the pathophysiology of cavernous malformations. J Neurol Neurosurg Psychiatry. 2001; 71:188-192. [PubMed: 11459890]

6. Toldo I, Drigo P, Mammi I, Marini V, Carollo C. Vertebral and spinal cavernous angiomas associated with familial cerebral cavernous malformation. Surg Neurol. 2008 
7. Liquori CL, et al. Mutations in a gene encoding a novel protein containing a phosphotyrosinebinding domain cause type 2 cerebral cavernous malformations. Am J Hum Genet. 2003; 73:14591464. [PubMed: 14624391]

8. Denier C, et al. Mutations within the MGC4607 Gene Cause Cerebral Cavernous Malformations. Am J Hum Genet. 2004; 74:326-337. [PubMed: 14740320]

9. Sahoo T, et al. Mutations in the gene encoding KRIT1, a Krev-1/rap1a binding protein, cause cerebral cavernous malformations (CCM1). Hum Mol Genet. 1999; 8:2325-2333. [PubMed: 10545614]

10. Laberge-le Couteulx S, et al. Truncating mutations in CCM1, encoding KRIT1, cause hereditary cavernous angiomas. Nat Genet. 1999; 23:189-193. [PubMed: 10508515]

11. Bergametti F, et al. Mutations within the Programmed Cell Death 10 Gene Cause Cerebral Cavernous Malformations. Am J Hum Genet. 2005; 76:42-51. [PubMed: 15543491]

12. Uhlik MT, et al. Rac-MEKK3-MKK3 scaffolding for p38 MAPK activation during hyperosmotic shock. Nat Cell Biol. 2003; 5:1104-1110. [PubMed: 14634666]

13. Petit N, Blecon A, Denier C, Tournier-Lasserve E. Patterns of expression of the three cerebral cavernous malformation (CCM) genes during embryonic and postnatal brain development. Gene Expr Patterns. 2006; 6:495-503. [PubMed: 16455310]

14. Plummer NW, et al. Neuronal expression of the $\mathrm{Ccm} 2$ gene in a new mouse model of cerebral cavernous malformations. Mamm Genome. 2006; 17:119-128. [PubMed: 16465592]

15. Seker A, et al. CCM2 expression parallels that of CCM1. Stroke; a journal of cerebral circulation. 2006; 37:518-523.

16. McCarty JH, et al. Selective ablation of \{alpha $\} \mathrm{v}$ integrins in the central nervous system leads to cerebral hemorrhage, seizures, axonal degeneration and premature death. Development. 2005; 132:165-176. [PubMed: 15576410]

17. Glading A, Han J, Stockton RA, Ginsberg MH. KRIT-1/CCM1 is a Rap1 effector that regulates endothelial cell cell junctions. J Cell Biol. 2007; 179:247-254. [PubMed: 17954608]

18. Risau W. Mechanisms of angiogenesis. Nature. 1997; 386:671-674. [PubMed: 9109485]

19. Su H, Mills AA, Wang X, Bradley A. A targeted X-linked CMV-Cre line. Genesis. 2002; 32:187188. [PubMed: 11857817]

20. Kisanuki YY, et al. Tie2-Cre transgenic mice: a new model for endothelial cell-lineage analysis in vivo. Dev Biol. 2001; 230:230-242. [PubMed: 11161575]

21. Sclafani AM, et al. Nestin-Cre mediated deletion of Pitx2 in the mouse. Genesis. 2006; 44:336344. [PubMed: 16823861]

22. Lepore JJ, et al. High-efficiency somatic mutagenesis in smooth muscle cells and cardiac myocytes in SM22alpha-Cre transgenic mice. Genesis. 2005; 41:179-184. [PubMed: 15789423]

23. Kamei M, et al. Endothelial tubes assemble from intracellular vacuoles in vivo. Nature. 2006; 442:453-456. [PubMed: 16799567]

24. Bayless KJ, Davis GE. Microtubule depolymerization rapidly collapses capillary tube networks in vitro and angiogenic vessels in vivo through the small GTPase Rho. J Biol Chem. 2004; 279:11686-11695. [PubMed: 14699132]

25. Wojciak-Stothard B, Potempa S, Eichholtz T, Ridley AJ. Rho and Rac but not Cdc42 regulate endothelial cell permeability. Journal of cell science. 2001; 114:1343-1355. [PubMed: 11257000]

26. Mohr C, Koch G, Just I, Aktories K. ADP-ribosylation by Clostridium botulinum C3 exoenzyme increases steady-state GTPase activities of recombinant rhoA and rhoB proteins. FEBS letters. 1992; 297:95-99. [PubMed: 1551445]

27. Hirose M, et al. Molecular dissection of the Rho-associated protein kinase (p160ROCK)-regulated neurite remodeling in neuroblastoma N1E-115 cells. J Cell Biol. 1998; 141:1625-1636. [PubMed: 9647654]

28. Kyriakis JM, Avruch J. Sounding the alarm: protein kinase cascades activated by stress and inflammation. J Biol Chem. 1996; 271:24313-24316. [PubMed: 8798679]

29. Jung KH, et al. Cerebral cavernous malformations with dynamic and progressive course: correlation study with vascular endothelial growth factor. Archives of neurology. 2003; 60:16131618. [PubMed: 14623736] 
30. Larson JJ, Ball WS, Bove KE, Crone KR, Tew JM Jr. Formation of intracerebral cavernous malformations after radiation treatment for central nervous system neoplasia in children. $\mathrm{J}$ Neurosurg. 1998; 88:51-56. [PubMed: 9420072]

31. Shi C, Shenkar R, Batjer HH, Check IJ, Awad IA. Oligoclonal immune response in cerebral cavernous malformations. Laboratory investigation. J Neurosurg. 2007; 107:1023-1026. [PubMed: 17977276]

32. Gault J, Shenkar R, Recksiek P, Awad IA. Biallelic somatic and germ line CCM1 truncating mutations in a cerebral cavernous malformation lesion. Stroke; a journal of cerebral circulation. $2005 ; 36: 872-874$.

33. Zeng L, et al. HMG CoA reductase inhibition modulates VEGF-induced endothelial cell hyperpermeability by preventing RhoA activation and myosin regulatory light chain phosphorylation. Faseb J. 2005; 19:1845-1847. [PubMed: 16160062]

34. Park HJ, et al. 3-hydroxy-3-methylglutaryl coenzyme A reductase inhibitors interfere with angiogenesis by inhibiting the geranylgeranylation of RhoA. Circ Res. 2002; 91:143-150. [PubMed: 12142347]

35. Kranenburg O, Poland M, Gebbink M, Oomen L, Moolenaar WH. Dissociation of LPA-induced cytoskeletal contraction from stress fiber formation by differential localization of RhoA. Journal of cell science. 1997; 110(Pt 19):2417-2427. [PubMed: 9410880]

36. Collisson EA, Carranza DC, Chen IY, Kolodney MS. Isoprenylation is necessary for the full invasive potential of RhoA overexpression in human melanoma cells. The Journal of investigative dermatology. 2002; 119:1172-1176. [PubMed: 12445208]

37. Im E, Kazlauskas A. Src family kinases promote vessel stability by antagonizing the Rho/ROCK pathway. J Biol Chem. 2007; 282:29122-29129. [PubMed: 17684019]

38. Mavria G, et al. ERK-MAPK signaling opposes Rho-kinase to promote endothelial cell survival and sprouting during angiogenesis. Cancer cell. 2006; 9:33-44. [PubMed: 16413470]

39. Whitehead KJ, Plummer NW, Adams JA, Marchuk DA, Li DY. Ccm1 is required for arterial morphogenesis: implications for the etiology of human cavernous malformations. Development. 2004; 131:1437-1448. [PubMed: 14993192]

40. Davis GE, Camarillo CW. An alpha 2 beta 1 integrin-dependent pinocytic mechanism involving intracellular vacuole formation and coalescence regulates capillary lumen and tube formation in three-dimensional collagen matrix. Experimental cell research. 1996; 224:39-51. [PubMed: 8612690]

41. Saunders WB, et al. Coregulation of vascular tube stabilization by endothelial cell TIMP-2 and pericyte TIMP-3. J Cell Biol. 2006; 175:179-191. [PubMed: 17030988]

42. Navankasattusas $\mathrm{S}$, et al. The netrin receptor UNC5B promotes angiogenesis in specific vascular beds. Development. 2008; 135:659-667. [PubMed: 18223200]

43. Saunders WB, Bayless KJ, Davis GE. MMP-1 activation by serine proteases and MMP-10 induces human capillary tubular network collapse and regression in 3D collagen matrices. Journal of cell science. 2005; 118:2325-2340. [PubMed: 15870107]

44. Nobes CD, Hall A. Rho GTPases control polarity, protrusion, and adhesion during cell movement. J Cell Biol. 1999; 144:1235-1244. [PubMed: 10087266] 

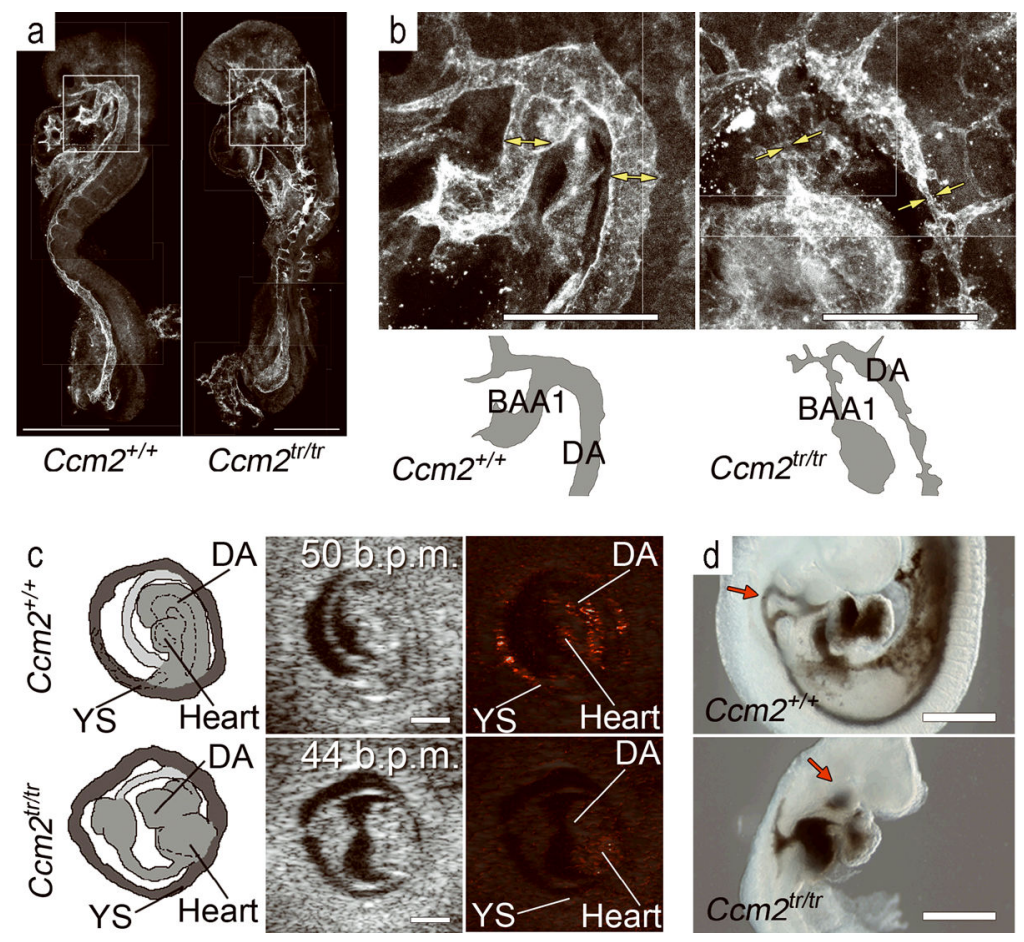

Figure 1. $C \mathrm{~cm} 2$ is required for circulation

(a) Whole mount confocal immunofluorescent micrographs of littermate embryos at E8.5 show abnormal vasculature in $\mathrm{Ccm} 2^{\text {tr/tr }}$ (right). (b) Higher magnification of the first branchial arch arteries (BAA1) and dorsal aorta (DA). See diagrams below for orientation. The wild type vessels (double arrows) are shown in contrast to the $\mathrm{Ccm} 2$ mutant, which (right) has a narrow endothelial connection at BAA1 and adjacent portions of the dorsal aorta (single arrows). (c) Fetal ultrasound demonstrates no flow in a $C c m 2^{\text {tr/tr }}$ embryo (bottom row) at E8.5 despite normal frequency of cardiac contractions (middle panel). Flow (colorized pixels) was seen in wild type littermates (top row). See diagrams (left panels) for orientation; DA - dorsal aorta, YS - yolk sac (also see Supplementary Movies 1 and 2). (d) Ink injection into the cardiac ventricle demonstrates flow through the branchial arch arteries at E9.5 in a wild type embryo (upper panel) with no anterograde flow through the branchial arches (red arrow) into the aorta in a $C \mathrm{~cm} 2^{t r / t r}$ littermate (lower panel). Scale bars: $500 \mu \mathrm{m}$ in (a), (c) and (d), and $200 \mu \mathrm{m}$ in (b). 


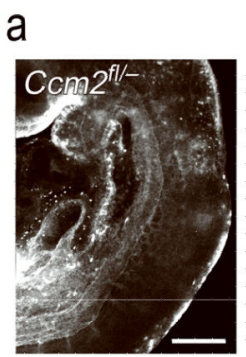

No CRE
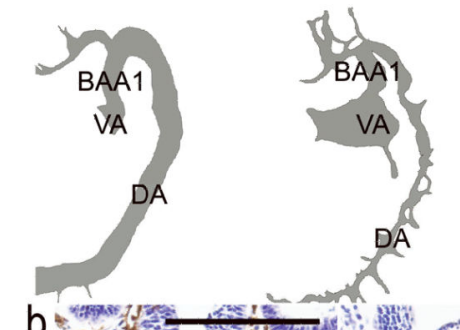

Endothelial

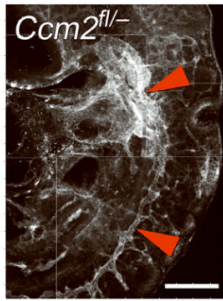

Tie2-CRE
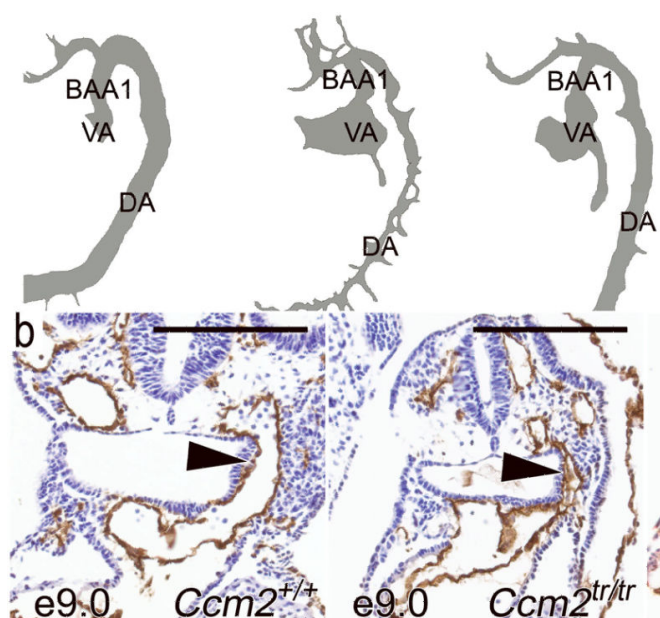

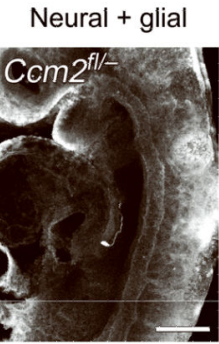

Nestin-CRE

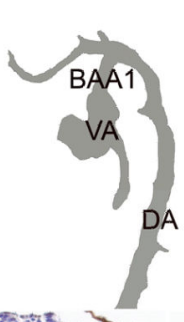

Smooth muscle

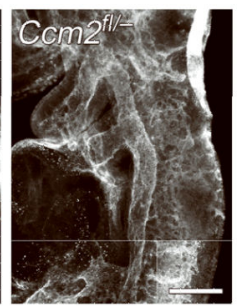

Tagin-CRE

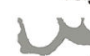

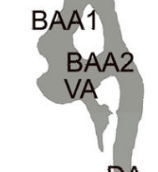
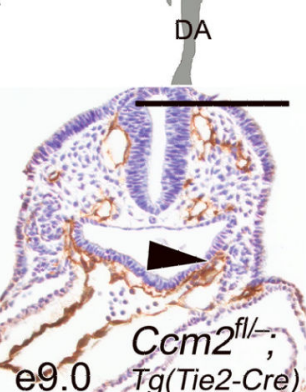

Figure 2. Vascular defects are endothelial autonomous

(a) Whole mount immunofluorescence demonstrates normal, uniform caliber branchial arch arteries and aortae in all $C \mathrm{~cm} 2^{\mathrm{fl} / \text { - }}$ embryos except the endothelial (Tie2-CRE) mutant, which has an irregular, narrow lumen (red arrowheads). Cartoons below are provided for orientation. BAA1 = first branchial arch artery, BAA2 = second branchial arch artery, VA= ventral aorta (or aortic sac), DA = dorsal aorta. (b) The narrow branchial arch arteries are well demonstrated on paraffin sections taken at E9.0. As opposed to the wild type embryo (left panel) the first branchial arch artery (arrowhead) is similarly narrowed and irregular in both the complete knockout $\left(\mathrm{Ccm} 2^{\mathrm{tr} / \mathrm{tr}}\right.$, middle panel) and the endothelial mutant $\left(C c m 2^{f l /-} ; T g(T i e 2-C R E)\right.$, right panel). Scale bars: $200 \mu \mathrm{m}$. 

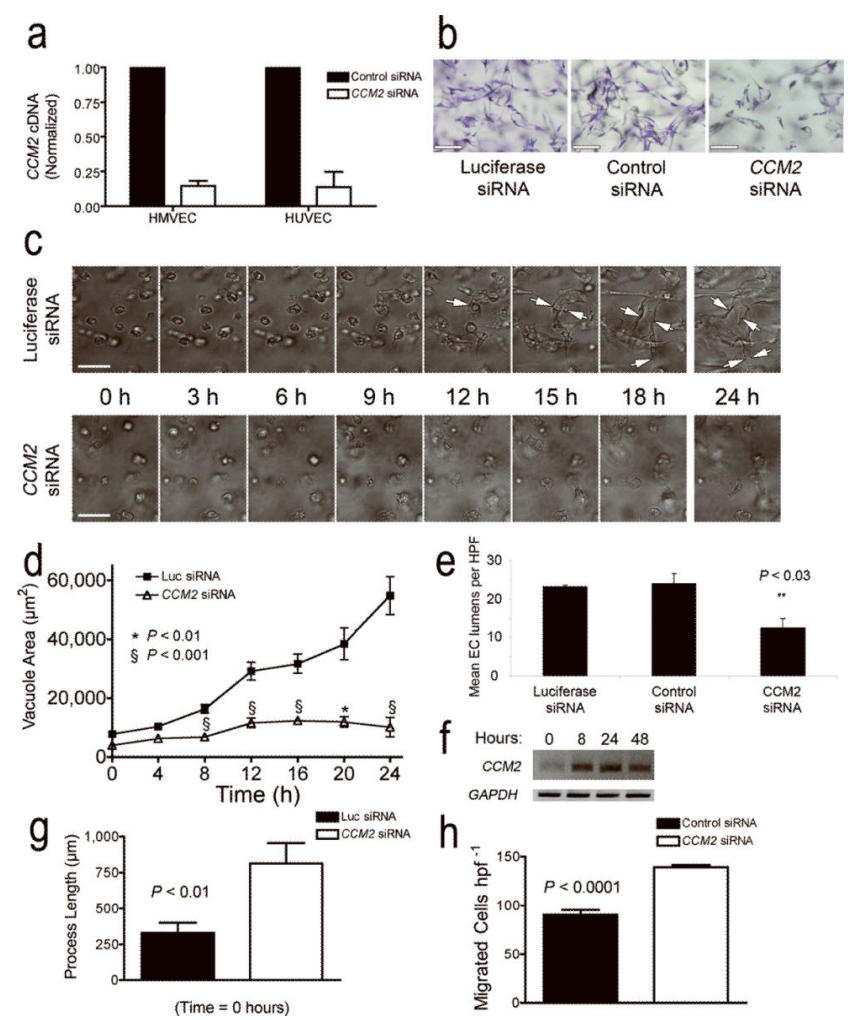

f Hours: $\begin{array}{lllll}0 & 8 & 24 & 48\end{array}$ ${ }_{\text {GAPDH }}^{C C M}---$

h.

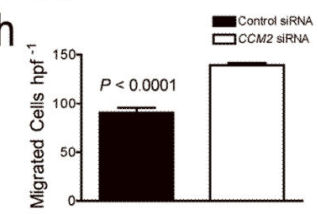

Figure 3. $C C M 2$ is required for endothelial tube morphogenesis

(a) CCM2 siRNA reduces the level of CCM2 transcripts in human dermal microvascular endothelial cells (HMVEC) and human umbilical vein endothelial cells (HUVEC) by $80 \%$.

(b) Treatment with CCM2 siRNA significantly reduces tube formation of HUVECs in threedimensional cultures in collagen. Two separate control siRNAs (luciferase siRNA, or a nonsense control siRNA) do not affect endothelial tube formation. (c) Time-lapse photography of tube development in endothelial cells treated with CCM2 siRNA compared with luciferase siRNAs. (d) Quantitation of lumen and vacuole development over time in HUVECs treated with CCM2 siRNA as compared to luciferase siRNA control. (e) Quantification of lumen numbers at $24 \mathrm{~h}$ in HUVECs treated with CCM2 siRNA compared to luciferase or non-sense controls. (f) RT PCR for CCM2 in control HUVECs undergoing tube-formation at various stages of the assay. (g) Quantification of filopodial length in HUVEC treated with CCM2 siRNA compared to luciferase siRNA control. (h) Haptotactic migration of HMVEC to fibronectin in CCM2-depleted cells versus non-sense siRNA control. Scale bars: $100 \mu \mathrm{m}$. Values are \pm s.e.m., except in (e) values are \pm s.d.. 


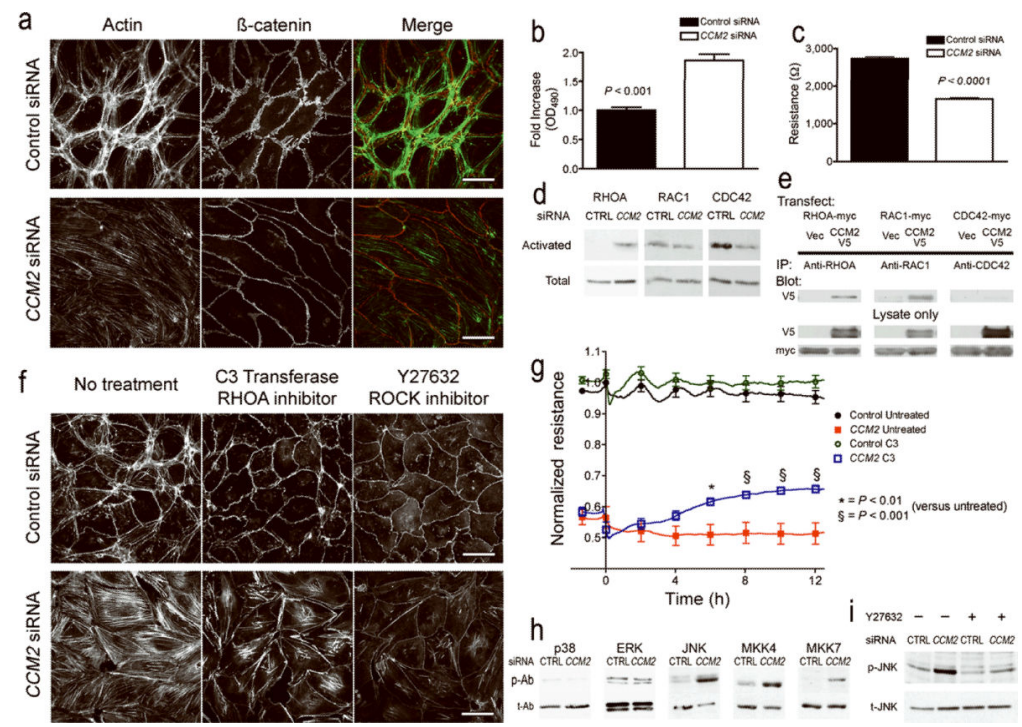

Figure 4. $C C M 2$ deficiency alters endothelial cytoskeletal architecture and cell-cell interaction via activation of the small GTPase RHOA

(a) Comparison of cellular cytoskeleton (actin fibers) and cell junctions ( $\beta$-catenin) in HMVEC treated with CCM2 or non-sense control siRNA. (b) Endothelial monolayer permeability to horseradish peroxidase (HRP) in HMVEC treated with CCM2 or non-sense control siRNA. (c) Decrease in transendothelial resistance in CCM2-depleted HMVECs compared to non-sense control. (d) Cells treated with CCM2 siRNA demonstrate increased active RHOA versus non-sense control siRNA, whereas there is little change in active RAC1, and a decrease in active CDC42. (e) CCM2 binds to RHOA and RAC1, but not CDC42. (f) Cytoskeletal changes with CCM2 siRNA are sensitive to inhibitors of Rho signaling. C3 transferase and Y-27632 both decrease cytoplasmic stress fibers in CCM2depleted HMVECs. (g) Treatment of HMVECs with C3 transferase improves the barrier function of CCM2-depleted cells but not control. (h) CCM2 deficiency activates the stress activated kinase, JNK and the JNK upstream kinases MKK4 and MKK7. p-Ab phosphorylated (active) kinase, t-Ab - total kinase. (i) Treatment of cells with Y-27632 decreases phosphorylated JNK (p-JNK) in CCM2-depleted HMVECs. Scale bars: $50 \mu \mathrm{m}$. Values are \pm s.e.m. 

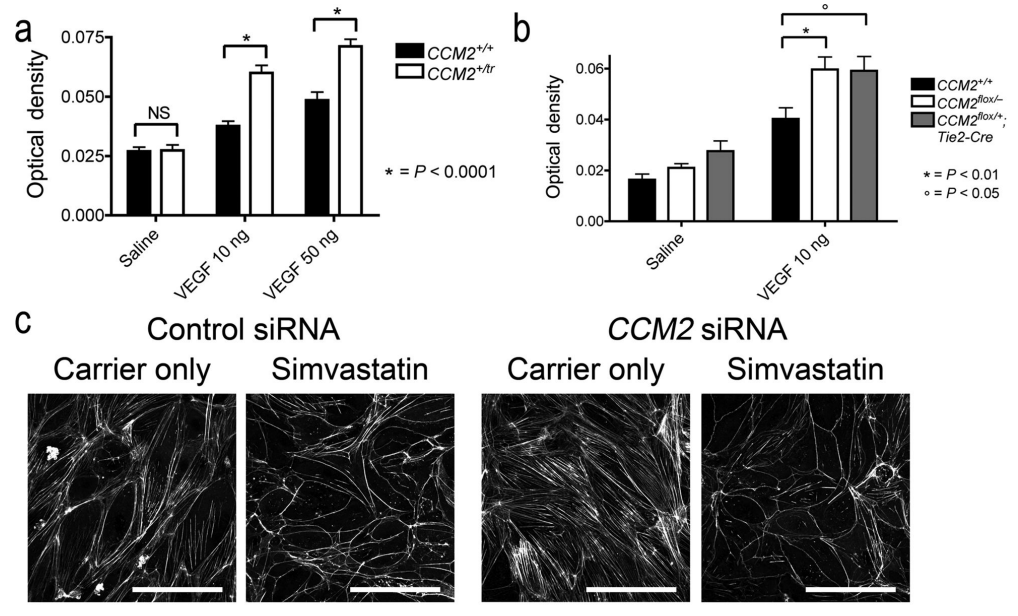

CCM2 SIRNA

d

Control siRnA

Carrier only

Simvastatin
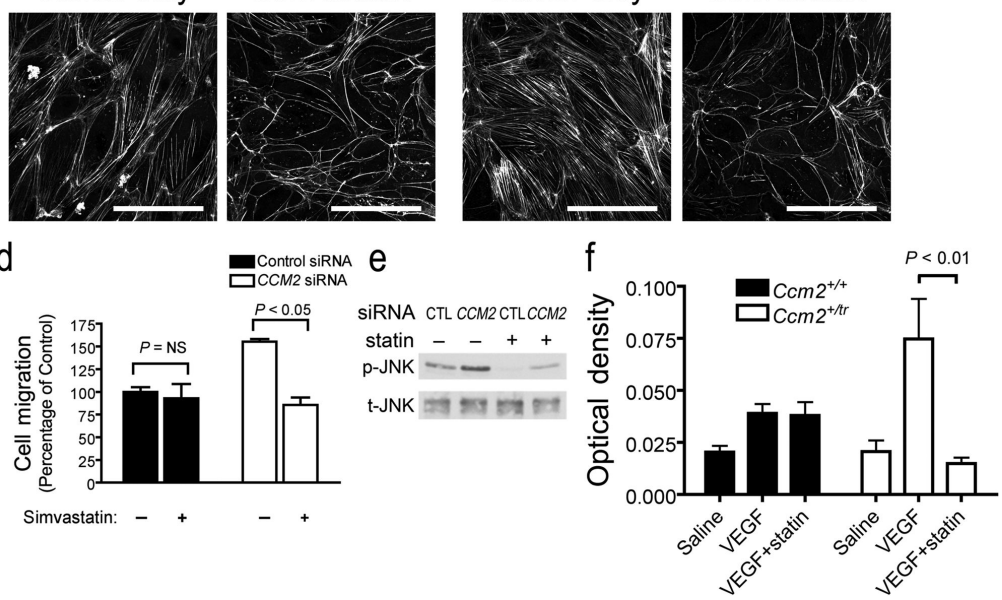

Figure 5. Heterozygous mice have permeability defects that can be rescued by treatment with Simvastatin

(a) $C \mathrm{~cm} 2^{+/ \mathrm{tr}}$ mice have increased vascular permeability in response to injected VEGF. The extravasation of Evans blue dye into the dermis was abnormally increased in $\mathrm{Ccm} 2^{+/ \mathrm{tr}}$ mice compared to $\mathrm{Ccm} 2^{+/+}$across a range of VEGF dosage. (b) Increased permeability is also observed in mice with endothelial specific heterozygosity for $\mathrm{Ccm} 2\left(\mathrm{Ccm} 2^{\mathrm{fl} /+} ; \mathrm{Tg}(\mathrm{Tie} 2-\right.$ $\mathrm{Cre}$ )). (c) Stress fibers are decreased in both control and CCM2-depleted HMVECs by treating the cells with simvastatin. (d) Simvastatin restores the migration response of CCM2depleted HMVECs back to the level of control. (e) Simvastatin also decreases the phosphorylation of JNK in both control and CCM2 siRNA treated HMVECs. (f) Pretreatment of mice with simvastatin decreased permeability in response to VEGF only in mice with mutations in $C c m 2$, not in wild type. Scale bars: $100 \mu \mathrm{m}$. Values are \pm s.e.m. 


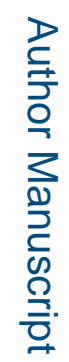

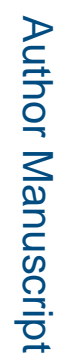

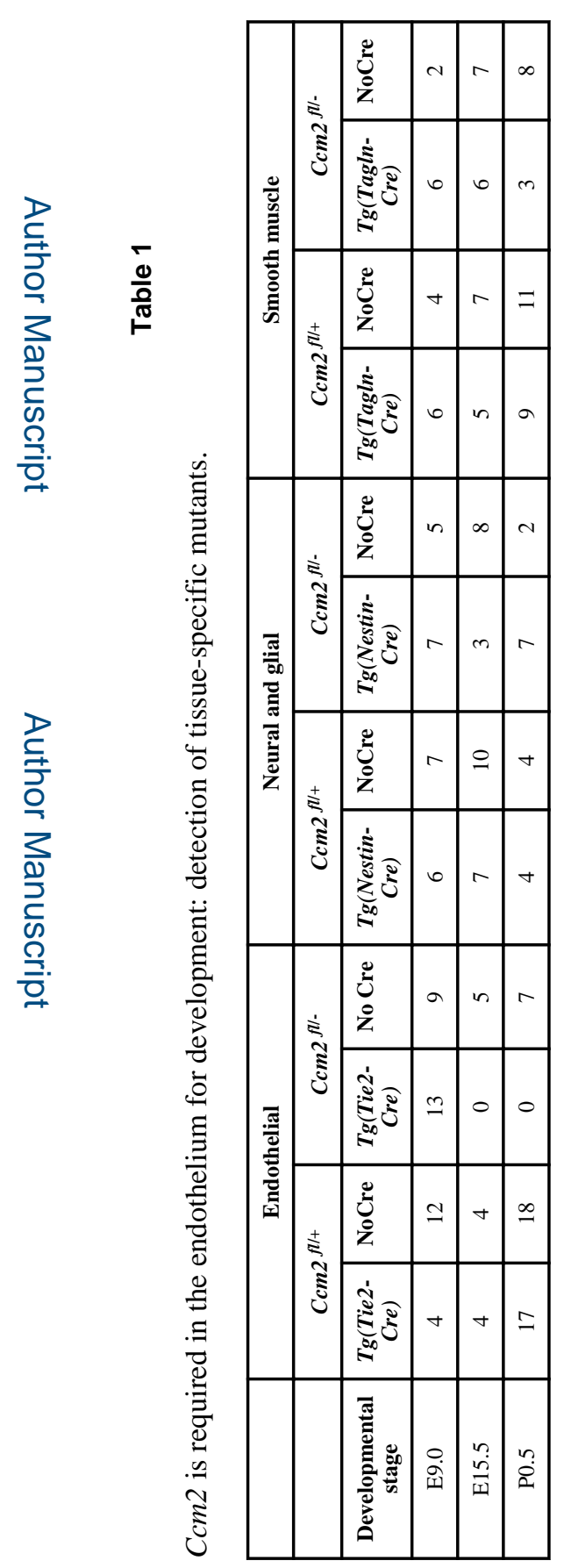

Nat Med. Author manuscript; available in PMC 2009 October 26. 